(2) Open Access Full Text Article

\title{
A prospective comparison of acute intestinal toxicity following whole pelvic versus small field intensity-modulated radiotherapy for prostate
}

\section{cancer}

Yeon Joo Kim

Jin-hong Park

In-Ha Yun

Young Seok Kim

Department of Radiation Oncology, Asan Medical Center, College of Medicine, University of Ulsan, Seoul, Republic of Korea
Correspondence: Young Seok Kim Department of Radiation Oncology, Asan Medical Center, College of Medicine, University of Ulsan, 88, Olympic-ro 43-gil, Songpa-gu, Seoul 05505, Republic of Korea

Tel +82230105614

Fax +82 230106950

Email ysk@amc.seoul.kr

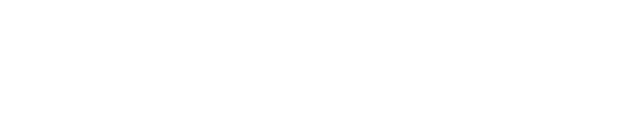

Purpose: To compare the acute intestinal toxicity of whole pelvic (WP) and small field (SF) intensity-modulated radiotherapy (IMRT) for prostate cancer using dosimetric and metabolic parameters as well as clinical findings.

Methods: Patients who received IMRT in either a definitive or postoperative setting were prospectively enrolled. Target volume and organs at risk including intestinal cavity (IC) were delineated in every patient by a single physician. The IC volume that received a $10-50$ Gy dose at 5-Gy intervals (V10-V50) and the percentage of irradiated volume as a fraction of total IC volume were calculated. Plasma citrulline levels, as an objective biological marker, were checked at three time points: baseline and after exposure to $30 \mathrm{~Gy}$ and $60 \mathrm{~Gy}$.

Results: Of the 41 patients, only six experienced grade 1 acute intestinal toxicity. Although all dose-volume parameters were significantly worse following WP than SF IMRT, there was no statistically significant relationship between these dosimetric parameters and clinical symptoms. Plasma citrulline levels did not show a serial decrease by radiotherapy volume difference (WP versus SF) and were not relevant to the irradiated doses.

Conclusion: Given that WP had comparable acute intestinal toxicities to those associated with SF, WP IMRT appears to be a feasible approach for the treatment of prostate cancer despite dosimetric disadvantages.

Keywords: prostate cancer, intensity-modulated radiotherapy, intestinal toxicity, citrulline

\section{Introduction}

The benefit of pelvic lymph node irradiation for intermediate- or high-risk prostate cancer remains controversial. Two prospective trials in a definitive setting have reported inconclusive results. The Radiation Therapy Oncology Group 9413 trial demonstrated better 4-year progression-free survival with whole pelvic (WP) compared with prostate-only radiotherapy (RT), although this benefit was no longer apparent 7 years after therapy. ${ }^{1,2}$ Another trial, GETUG-01, reported no difference in 5-year progression-free survival between WP and prostate-only RT. ${ }^{3}$ However, GETUG-01 study has been criticized for its low superior border, S1/S2 interspace. Several retrospective studies have also consistently suggested the efficacy of WP irradiation. ${ }^{4-6}$ Likewise, in a postoperative setting, a few retrospective trials showed the efficacy of pelvic nodal irradiation. ${ }^{7,8}$

Radiation-related toxicities are major concerns for the broad adoption of pelvic RT, and small bowel is the one of the dose-limiting organs. Several studies have 
reported that acute bowel complications were higher in patients treated with whole pelvic than in those receiving prostate-only RT, ${ }^{4,9}$ but with small insignificant increases in late intestinal toxicity. ${ }^{1,3}$ Most of these studies were based on three-dimensional conformal RT. Recent dosimetric studies of patients who had received WP RT using intensity-modulated radiotherapy (IMRT) revealed that IMRT significantly reduced the volume irradiated to pelvic organs, such as the rectum and small bowel. ${ }^{10-14}$ Accordingly, acute gastrointestinal (GI) toxicities were reduced with the use of IMRT. ${ }^{11,15,16}$

Attempts to define the dosimetric parameters of intestinal toxicities indicated either that the most predictive parameter was $\mathrm{V} 45^{17}$ or that V45, V50, and V55 were best predictors of dose-volume relationships in patients with prostate cancer. ${ }^{18}$ The most significant factor correlating with acute small bowel toxicity in gynecology patients undergoing WP RT was V35. ${ }^{15}$ In anal cancer, V30 correlated with acute intestinal toxicities. ${ }^{19}$

Citrulline, the metabolic end product of the metabolism of glutamine by enterocytes, is a reliable biomarker of functioning enterocyte mass that reflects intestinal damage. ${ }^{20-22}$ Injury to the small intestine could lead to decline in circulating citrulline. Based on this phenomenon, Onal et $\mathrm{al}^{23}$ reported that decrement of plasma citrulline levels could predict intestinal toxicity in patients treated with pelvic RT. A citrulline-based assessment of radiation-induced intestinal toxicity might provide an objective method to measure and monitor intestinal toxicity during and after pelvic RT.

The purpose of this study was to compare acute bowel toxicity between WP and small field (SF) in patients with prostate cancer treated with IMRT. We also evaluated the dosimetric parameters and plasma citrulline levels as potential objective markers of the acute intestinal toxicity.

\section{Materials and methods}

A total of 49 consecutive patients with prostate cancer who received definitive or postoperative RT were enrolled prospectively. All patients provided written informed consent, and the study was approved by the institutional review board (Asan Medical Center, approval number 2013-0160). The patients were treated with one of three aims of RT: 1) definitive aim, 2) adjuvant aim, which means RT within 4-8 weeks after the prostatectomy without detectable prostate specific antigen (PSA), 3) salvage aim defined as RT after biochemical relapse, which is an elevation of PSA $\geq 0.2 \mathrm{ng} / \mathrm{mL}$ followed by another increase.
A planning computed tomography (CT) scan with a slice thickness of $2.5 \mathrm{~mm}$ was performed in the supine position with ankle immobilization. The scan region ranged from the second lumbar vertebrae to the proximal one-third of the femur. Each patient was instructed to empty their bowel and bladder immediately before simulation and all subsequent treatment sessions. An endorectal balloon was used to restrict the irradiated volume to the rectum and for precise immobilization of the prostate or prostate bed.

In the case of definitive SF IMRT, the gross target volume (GTV) included the whole prostate and the clinical target volume (CTV) included the GTV with or without seminal vesicles, depending on the clinical situation. For WP IMRT, GTV included the whole prostate and the involved lymph nodes if any. The CTV included the GTV, seminal vesicles, and internal iliac, external iliac, and obturator nodal regions. The upper boarder of the CTV was the level of the common iliac bifurcation, which was generally located at or immediately above the L5/S1 junction. Seminal vesicles were not included in CTV for low-risk group patients. For high-risk patients, without evidence of invasion of distal seminal vesicle, only the proximal $2.0-2.5 \mathrm{~cm}$ was included within CTV according to Kestin et al. ${ }^{24}$ In postoperative setting, the bed of prostate with or without seminal vesicle bed was covered for SF IMRT patients, while the internal iliac, external iliac, and obturator nodal regions were also covered for WP IMRT patients. Patients who experienced the surgery had more precise information on seminal vesicle invasion, so we included the seminal vesicle bed for those who had pathologically seminal vesicle invasion only. The planning target volume (PTV) was a $3 \mathrm{~mm}$ expansion posteriorly and 5-7 $\mathrm{mm}$ expansion for the remainders of the CTV. After pelvic RT, the boost treatment included only the GTV, seminal vesicles, and metastatic lymph nodal regions. For dosimetric analysis, the intestinal cavity (IC) was contoured by one author (YJ Kim) according to the IC definition provided by Sanguineti et al. ${ }^{25} \mathrm{We}$ calculated the IC volume that received a 10-50 Gy dose at 5-Gy intervals (V10-V50) and the percentage of irradiated IC volume as a fraction of total IC volume. Cone-beam computed tomography (CBCT) guidance was performed daily to improve the setup stability. Details of the image guidance protocol are as follows. Before each CBCT scan, electronic portal imaging (guided by skin marks) was performed to ensure proper positioning and inflation of the endorectal balloon. And manual or automatic registration of the planning CT and each CBCT was performed, based on the bony anatomy of the pelvis. Then, manual matching 
was conducted based on the prostate or anterior rectal wall in cases of postprostatectomy RT.

All patients were treated with the fraction size of $2.2 \mathrm{~Gy}$. In the adjuvant or salvage aim, WP doses were 44 Gy in 20 fractions and boost doses that excluded elective nodal irradiation were 66 Gy in 30 fractions. In definitive aim, total boost doses were up to 72.6 Gy in 33 fractions. IMRT schemes using five to seven fields were created using an Eclipse 10.0 instrument (Varian Medical Systems, CA, USA). Dose constraints were as follows: 1) for PTV, $>95 \%$ PTV receives equal to higher than the prescribed dose; 2) for rectum, V70 $<20 \%$ and V60 <35\%; 3) for bladder, V60 <35\%; and 4) for penile bulb, mean dose $<52.5 \mathrm{~Gy}$.

Assessments of clinical toxicities were done weekly during the treatment period and at 3 months after RT. Acute complications were defined as occurring within 3 months after treatment. Toxicities were recorded using the National Cancer Institute's Common Terminology Criteria for Adverse Events, Version 4.02. Plasma citrulline levels were assessed three times: at baseline, after exposure to $30 \mathrm{~Gy}$, and after exposure to $60 \mathrm{~Gy}$ to target volume. Blood samples were collected $\sim 15$ minutes before irradiation. Before blood samples were collected, patients were instructed to fast for at least 6 hours and to sit down for 15 minutes while avoiding any physical exertion. Thereafter, $1.5 \mathrm{~mL}$ of blood was taken and collected in an ethylenediaminetetraacetic acid bottle. Within 3 hours, plasma was obtained through whole blood configuration at $3,000 \mathrm{rpm}$ for 10 minutes at $4^{\circ} \mathrm{C}$, and $700 \mu \mathrm{L}$ of plasma was stored at $-70^{\circ} \mathrm{C}$ until further processing.
A total of 123 samples from 41 patients were sent to a commercial laboratory (Seoul Medical Science Institute, Seoul, Korea) to measure the plasma citrulline levels. Thereafter, $30 \mu \mathrm{L}$ of $30 \%$ sulfosalicylic acid was added to $300 \mu \mathrm{L}$ plasma for deproteinization, and ion-exchange high-performance liquid chromatography was used to measure the plasma citrulline concentration $(\mu \mathrm{mol} / \mathrm{L})$.

Mann-Whitney $U$-tests were used to detect statistically significant differences between the WP and SF IMRT groups in terms of patient characteristics, acute intestinal toxicities, and volumes receiving 10-50 Gy in 5-Gy intervals for ICs. Acute intestinal toxicity was expressed as a crude rate. Spearman's correlation was used to test correlations between acute intestinal toxicity, dose-volume, and citrulline concentration. A correlation was considered to be good if the value of Spearman's correlation factor was $\geq 0.7$. A correlation was considered moderate if the value of Spearman's correlation factor was $0.5-0.7$ and poor at values $<0.5$. One-way analysis of variance test was used to assess the relationship between dose and volume for plasma citrulline and clinical toxicity. All statistical analyses were performed using SPSS software Version 21.0 (IBM Corporation, Armonk, NY, USA).

\section{Results}

Of the 49 patients enrolled in this study, eight were excluded; six patients who refused RT, one patient who requested treatment at another hospital, and one patient owing to the failure to analyze a sample of plasma citrulline. The characteristics of the 41 patients analyzed are listed in Table 1. Median

Table I Patient characteristics

\begin{tabular}{|c|c|c|c|}
\hline Characteristics & WP & SF & $P$-value \\
\hline Number of patients & 29 & 12 & \\
\hline Age (years), median (range) & $69(50-83)$ & $69(49-78)$ & 0.62 \\
\hline Follow-up (months), median (range) & $6(3-9)$ & $7(3-11)$ & 0.04 \\
\hline Risk group, n (\%) & & & 0.02 \\
\hline Low & 0 & 0 & \\
\hline Intermediate & $4(14)$ & $7(58)$ & \\
\hline High & $25(86)$ & $5(42)$ & \\
\hline Aim of RT, n (\%) & & & 0.83 \\
\hline Definitive & $9(31)$ & $4(33)$ & \\
\hline Salvage & $19(66)$ & $8(67)$ & \\
\hline Adjuvant & I (3) & & \\
\hline Concurrent ADT, n (\%) & & & 0.04 \\
\hline Yes & $24(83)$ & $4(33)$ & \\
\hline No & $5(7)$ & $8(67)$ & \\
\hline Intestinal volume (cc), median (range) & I,I54 (539.7-I,79I.7) & I,290.9 (727.9-I,482.3) & 0.52 \\
\hline CTV (cc), median (range) & $314.2(258.2-465.1)$ & $51.5(31.6-97.5)$ & 0.14 \\
\hline PTV (cc), median (range) & $572(475 . \mid-80 I .6)$ & $94.9(62.2-152.1)$ & 0.92 \\
\hline
\end{tabular}

Abbreviations: ADT, androgen deprivation therapy; CTV, clinical target volume; PTV, planning target volume; RT, radiotherapy; SF, small field; WP, whole pelvic. 
Table 2 Grade I acute gastrointestinal toxicities following WP and SF IMRT

\begin{tabular}{|c|c|c|c|c|c|c|c|}
\hline \multirow{2}{*}{$\begin{array}{l}\text { Intestinal } \\
\text { toxicities }\end{array}$} & \multicolumn{2}{|c|}{ Number of events (\%) } & \multirow[t]{2}{*}{$P$-value } & \multirow{2}{*}{$\begin{array}{l}\text { Rectal } \\
\text { toxicities }\end{array}$} & \multicolumn{2}{|c|}{ Number of events (\%) } & \multirow[t]{2}{*}{$P$-value } \\
\hline & WP & SF & & & WP & SF & \\
\hline Diarrhea $^{\mathrm{a}}$ & $2(7)$ & $I(8)$ & 0.66 & Proctitis $^{\mathrm{a}}$ & $4(14)$ & I (8) & 0.29 \\
\hline Anorexia $^{a}$ & I (3) & 0 & & & & & \\
\hline Dyspepsia $^{a}$ & $2(7)$ & 0 & & & & & \\
\hline Total & $5(17)$ & I (8) & & & & & \\
\hline
\end{tabular}

Note: aAll toxicities were grade I.

Abbreviations: IMRT, intensity-modulated radiotherapy; SF, small field; WP, whole pelvic.

follow-up time was 6 months (range: $3-11$ months). According to our center protocol, patients with Roach score $\geq 15 \%{ }^{26}$ were treated with WP IMRT, and the other patients had SF IMRT. Of the 29 patients in the WP group, nine received definitive IMRT and 19 were treated with salvage IMRT. Only one individual received adjuvant IMRT. Among 12 patients who received SF IMRT, four were treated with definitive aim and the remaining with a salvage aim.

Acute GI toxicities are listed in Table 2. In case of intestinal toxicities, IMRT was tolerated well regardless of field size, with only six events of grade 1 acute toxicities. Five events $(17 \%)$ were observed for the WP group and only one event (8\%) for the SF group. The symptoms of intestinal toxicity were diarrhea $(n=2)$, anorexia $(n=1)$, and dyspepsia $(n=2)$. One of the patients who received definitive SF IMRT suffered from grade 1 dyspepsia and abdominal pain. Overall, there were no significant differences in acute intestinal toxicity based on clinical criteria between the WP and SF IMRT groups $(P=0.66)$. We also reviewed proctitis for comparing GI toxicities to those of previous studies; only four patients (14\%) in WP group suffered from grade 1 proctitis. Only one patient (8\%) in SF group experienced grade 1 proctitis. Table 3 lists the threshold volumes calculated. All ranges of dose-volume parameters were significantly higher for the WP IMRT than those of the SF IMRT group. Owing to low rate of overall acute intestinal toxicities in our present cohort, we could not find the most appropriate $V_{\text {dose }}$ parameter to predict radiation-induced toxicity of IC.

No significant decrease in the plasma citrulline level was apparent during the treatment (Table 4). There was no difference in plasma citrulline concentration between patients of the WP and SF IMRT groups. No significant correlations were observed between the plasma citrulline level and either the radiation dose or the clinical intestinal toxicity. Even in the patients with grade 1 intestinal toxicities, although two patients showed ultimate citrulline drop after irradiation of $60 \mathrm{~Gy}$, the others presented the elevation of citrulline levels (Table 5).

\section{Discussion}

The primary objective of this study was to compare acute intestinal toxicities after WP and SF IMRT in patients with prostate cancer. We also set out to evaluate dose-volume parameters between these two treatment groups. All dosevolume parameters were significantly higher in WP group compared with the SF IMRT group. However, mild acute intestinal toxicity (grade 1) occurred in only six of our patients, five in the WP and one in the SF group ( $P=0.66$ ). Despite including the incidence of proctitis, the frequency of acute intestinal toxicity of ours was lower than other published studies. ${ }^{27,28}$

Table 3 Threshold volumes of small intestine following WP and SF IMRT

\begin{tabular}{|c|c|c|c|c|c|c|}
\hline \multirow{2}{*}{$\begin{array}{l}\text { Threshold } \\
\text { volumes }^{\mathrm{a}}\end{array}$} & \multicolumn{2}{|c|}{ Absolute volume (cc), (mean \pm SD) } & \multirow[t]{2}{*}{$P$-value } & \multicolumn{2}{|c|}{ Percentage (mean \pm SD) } & \multirow[t]{2}{*}{$P$-value } \\
\hline & WP & SF & & WP & SF & \\
\hline VI0 & $763.5 \pm 243.8$ & $35.4 \pm 33.9$ & $<0.01$ & $66.0 \pm 8.5$ & $3.0 \pm 2.9$ & $<0.01$ \\
\hline VI5 & $411.9 \pm 225.9$ & $27.4 \pm 26.7$ & $<0.01$ & $61.8 \pm 9.2$ & $2.3 \pm 2.2$ & $<0.01$ \\
\hline V20 & $608.6 \pm 187.0$ & $18.3 \pm 20.8$ & $<0.01$ & $53.3 \pm 9.4$ & $1.5 \pm 1.7$ & 0.01 \\
\hline V25 & $439.8 \pm 134.5$ & $13.8 \pm 15.8$ & $<0.01$ & $38.9 \pm 8.7$ & $1.1 \pm 1.3$ & $<0.01$ \\
\hline V30 & $321.9 \pm 103.7$ & $8.4 \pm 11.6$ & $<0.01$ & $27.7 \pm 7.1$ & $0.7 \pm 0.9$ & $<0.01$ \\
\hline V35 & $232.7 \pm 81.0$ & $3.1 \pm 8.8$ & $<0.01$ & $20.5 \pm 5.5$ & $0.5 \pm 0.7$ & $<0.01$ \\
\hline V40 & $167.6 \pm 63.6$ & $3.7 \pm 6.1$ & $<0.01$ & $14.7 \pm 4.2$ & $0.3 \pm 0.5$ & $<0.01$ \\
\hline V45 & $86.4 \pm 49.5$ & $2.7 \pm 4.4$ & $<0.01$ & $7.4 \pm 3.3$ & $0.2 \pm 0.3$ & $<0.01$ \\
\hline V50 & $13.3 \pm 18.9$ & $1.6 \pm 2.8$ & $<0.01$ & $1.0 \pm 1.3$ & $0.1 \pm 0.2$ & $<0.01$ \\
\hline
\end{tabular}

Note: ${ }^{\vee} \vee x$ indicates volume of intestinal cavity receiving at least $x$ Gy.

Abbreviations: IMRT, intensity-modulated radiotherapy; SF, small field; WP, whole pelvic. 
Table 4 Citrulline levels $(\mathrm{ng} / \mathrm{mL})$ at baseline, and after irradiating at $30 \mathrm{~Gy}$ and $60 \mathrm{~Gy}$

\begin{tabular}{lllll}
\hline Group & Baseline & At 30 Gy & At 60 Gy & P-value \\
\hline Total & $30.6 \pm 12.7$ & $34.1 \pm 20.1$ & $32.4 \pm 16.1$ & 0.61 \\
WP & $30.9 \pm 13.6$ & $38.2 \pm 21.8$ & $31.6 \pm 16.0$ & 0.95 \\
SF & $30.1 \pm 10.5$ & $24.1 \pm 10.5$ & $34.4 \pm 16.7$ & 0.66 \\
P-value & 0.60 & 0.13 & 0.83 & \\
\hline
\end{tabular}

Note: Data shown as mean \pm SD or $P$-value.

Abbreviations: SF, small field; WP, whole pelvic.

A first reason for this might be the relatively small target volume we delineated. Unlike CTV in Deville et al ${ }^{27}$ which always contained the seminal vesicles, we selectively included them. The seminal vesicles are the key organs for deciding upper margin of CTV in SF group and for reducing CTV volume of WP group. We also used small PTV margin, $3 \mathrm{~mm}$ expansion posteriorly and 5-7 $\mathrm{mm}$ expansion for the rest of the CTV. It was possible owing to improvement of setup stability through the daily CBCT guide and the use of rectal balloon since rectal balloons were easily seen in the CBCT. Second, the number of patients enrolled was too small. Regardless, the results of the current analysis showed that WP IMRT is not inferior to SF IMRT in terms of causing acute intestinal toxicity. Supporting the assumption, WP IMRT group in this study contained more postoperative RT patients who were expected to have higher intestinal toxicities. ${ }^{29}$

Some might concern about the effect of androgen deprivation therapy (ADT) to GI toxicities. Most patients ( $\mathrm{n}=24$, 83\%) in the WP group had ADT concurrently with RT, while only four patients (33\%) in the SF group were treated with concurrent RT and ADT. There was no report about GI toxicities originated from ADT. Recently, a study reported the long-term quality-of-life outcomes in patients with locally advanced prostate cancer using the European Organization for Research and Treatment of Cancer (EORTC) Core Questionnaire that had a section for bowel symptoms. ${ }^{30}$ They enrolled 1,205 patients and randomly allocated them to either ADT alone or with RT. Baseline score of EORTC bowel symptoms was 3.6 before ADT, and after 6-month, 12-month, and 36-month follow-up, the scores were not elevated, which

Table 5 Citrulline levels $(\mathrm{ng} / \mathrm{mL})$ of patients with grade I intestinal toxicities

\begin{tabular}{llll}
\hline Case & Baseline & At 30 Gy & At 60 Gy \\
\hline 1 & 28.5 & 48.3 & 52.6 \\
2 & 31.5 & 24.5 & 59.1 \\
3 & 44.1 & 17.7 & 47.3 \\
4 & 36.8 & 50.1 & 6.1 \\
5 & 24.5 & 30.6 & 12.7 \\
\hline
\end{tabular}

means that the toxicities were not increased. Excluding the influence of ADT to intestinal toxicities through Brundage et al, ${ }^{30}$ WP IMRT showed similar acute intestinal toxicities (Table 1). We thus speculate, although with caution, that WP IMRT might be an acceptable therapy for patients with prostate cancer at a high risk of pelvic LN metastasis with good tolerability.

Moreover, recently, Harris et al ${ }^{31}$ reported a modification of pelvic lymph node contouring smaller than Radiation Therapy Oncology Group guideline. With the new delineation guideline expected to lead to less toxicities without compromising treatment outcomes, they launched the prostate and pelvis versus prostate-alone treatment for locally advanced prostate cancer (PIVOTAL) Phase II trial of prostate-alone versus prostate and pelvic node IMRT (ISRCTN 48709247). Through the efforts like this, we anticipate that more patients could get benefit from WPRT with no fear of toxicities.

Deville et $\mathrm{al}^{27}$ reported clinical toxicities and dosimetric parameters after WP and SF IMRT for prostate cancer in both definitive and postoperative settings ${ }^{28}$ In their definitive setting study, grade 1 or 2 acute GI toxicity occurred in $70 \%$ of patients treated with WP IMRT, whereas $37 \%$ of the SF IMRT patients reported acute bowel toxicity $(P=0.01)$. Similar to their observations for the postoperative setting, $92 \%$ of WP IMRT and $49 \%$ of SF IMRT patients showed grade 1 or 2 acute GI toxicity $(P=0.01)$. There were no instances where acute intestinal toxicity was grade 3 or higher. Since these authors contoured the bowel only in the WP IMRT group in their definitive study, ${ }^{27}$ dosimetric comparisons with our current analyses for IC values were not possible. Perna et $\mathrm{al}^{18}$ investigated the correlation between clinical dosimetric parameters and acute bowel toxicity. Among their 96 patients with prostate cancer who received WP IMRT, 15 (15.6\%) experienced grade 2 toxicity, and the best dosevolume predictors were V45, V50, and V55 (V45 $\geq 50 \mathrm{cc}$, V50 $\geq 13 \mathrm{cc}, \mathrm{V} 55 \geq 3 \mathrm{cc} ; P$-values, $0.01-0.03)$. Fiorino et al ${ }^{17}$ conducted the same study in patients with prostate cancer treated with WP RT using both a conventional four-field technique and an IMRT. Univariate analyses in that report showed a significant correlation between V20-V50 and toxicity $(P<0.01)$, with a higher predictive value observed for V40-V50 than for V20-V50. Multivariate analysis showed that the most predictive dosimetric parameter was V45 $(P=0.01)$.

The other parameter we tried to evaluate in our present analysis was the plasma citrulline level. Citrulline is a metabolic end product of small bowel enterocytes and has been proposed as a noninvasive marker for intestinal damage. 
Crenn et $\mathrm{a}^{32}$ assessed the plasma citrulline concentration in patients with prostate cancer with nonmalignant short bowel syndrome and reported that citrulline levels were significantly lower in patients with short bowel syndrome than in control patients $(P<0.01)$. Other studies have also reported the clinical use of citrulline as a biomarker for intestinal toxicity related to chronic villous atrophy, chemotherapy, and transplantation of the small bowel. ${ }^{20}$ Lutgens et a ${ }^{33}$ reported that plasma citrulline concentrations correlated with clinical toxicity during fractionated RT of 23 patients with prostate cancer $(P<0.001)$. This showed the feasibility of plasma citrulline as a marker for radiation-induced intestinal toxicity. Analysis of 53 patients with prostate cancer by Onal et al ${ }^{23}$ also indicated that plasma citrulline levels could predict intestinal toxicity in pelvic RT. They classified patients into three groups in terms of changes in citrulline concentrations: 1) $<20 \%$, 2) $21 \%-40 \%$, and 3) $>40 \%$. V5-V45 values were significantly different between the three groups.

As Onal et al failed to provide the exact volume of all dose-volume values, we could not conduct a direct comparison of their reported dose-volume parameters and those of this study. However, their analysis of the dose-volume histogram graphs of each treatment group indicates that the V45 and V50 in group 1 in that study, for which citrulline levels showed only a minimal change, were similar to the V45 and V50 values of WP IMRT group in this study (V45: $8 \%$ versus $7 \%$; V50\%: $2 \%$ versus $1 \%$ ). Given that previous studies noted that V45-V50 were the best dose-volume predictors for acute intestinal toxicity, ${ }^{17,18}$ the low incidence of intestinal toxicity in this study could be explained by the low volume of the area that received a high dose of irradiation. Onal et al used four-field box technique so that most small bowel segments were within RT field, while this study utilized IMRT for small bowel sparing. This contributed to the failure to detect a significant decrease in the level of plasma citrulline. To validate the feasibility of plasma citrulline level in use of IMRT, larger field than WP RT would be needed such as extended field RT in cervical cancer.

As mentioned previously, the limitations of this study were 1) the small number of patients included and experienced significant bowel toxicity, 2) owing to the short follow-up period, late intestinal toxicity could not be assessed, and 3) not large RT fields enough to show the relation of clinical intestinal toxicities and plasma citrulline levels. With larger radiation volumes, such as extended field RT in the treatment of cervical cancer, we may prove the usefulness of plasma citrulline as a predictive factor for intestinal toxicity.

\section{Conclusion}

Present analyses do demonstrate that WP IMRT does not produce poorer outcomes in terms of acute intestinal toxicity than SF IMRT, suggesting the feasibility of WP IMRT. The very low incidence of acute intestinal toxicity prevented us from definitively testing the usefulness of dosimetric and metabolic parameters. We need to wait for further large prospective trials, including PIVOTAL trial.

\section{Acknowledgment}

This study was supported by a grant (2013-0160) from the Asan Institute for Life Science, Seoul, Korea.

\section{Disclosure}

The authors report no conflicts of interest in this work.

\section{References}

1. Roach M 3rd, DeSilvio M, Lawton C, et al; Radiation Therapy Oncology Group 9413. Phase III trial comparing whole-pelvic versus prostateonly radiotherapy and neoadjuvant versus adjuvant combined androgen suppression: Radiation Therapy Oncology Group 9413. J Clin Oncol. 2003;21(10):1904-1911.

2. Lawton CA, DeSilvio M, Roach M 3rd, et al. An update of the phase III trial comparing whole pelvic to prostate only radiotherapy and neoadjuvant to adjuvant total androgen suppression: updated analysis of RTOG 94-13, with emphasis on unexpected hormone/radiation interactions. Int J Radiat Oncol Biol Phys. 2007;69(3):646-655.

3. Pommier P, Chabaud S, Lagrange JL, et al. Is there a role for pelvic irradiation in localized prostate adenocarcinoma? Preliminary results of GETUG-01. J Clin Oncol. 2007;25(34):5366-5373.

4. Aizer AA, Yu JB, McKeon AM, Decker RH, Colberg JW, Peschel RE. Whole pelvic radiotherapy versus prostate only radiotherapy in the management of locally advanced or aggressive prostate adenocarcinoma. Int J Radiat Oncol Biol Phys. 2009;75(5):1344-1349.

5. Pan CC, Kim KY, Taylor JM, McLaughlin PW, Sandler HM. Influence of 3D-CRT pelvic irradiation on outcome in prostate cancer treated with external beam radiotherapy. Int J Radiat Oncol Biol Phys. 2002;53(5):1139-1145.

6. Seaward SA, Weinberg V, Lewis P, Leigh B, Phillips TL, Roach M 3rd. Improved freedom from PSA failure with whole pelvic irradiation for high-risk prostate cancer. Int J Radiat Oncol Biol Phys. 1998;42(5): $1055-1062$.

7. Spiotto MT, Hancock SL, King CR. Radiotherapy after prostatectomy: improved biochemical relapse-free survival with whole pelvic compared with prostate bed only for high-risk patients. Int J Radiat Oncol Biol Phys. 2007;69(1):54-61.

8. Kim BS, Lashkari A, Vongtama R, Lee SP, Parker RG. Effect of pelvic lymph node irradiation in salvage therapy for patients with prostate cancer with a biochemical relapse following radical prostatectomy. Clin Prostate Cancer. 2004;3(2):93-97.

9. Mameghan H, Fisher R, Mameghan J, Watt WH, Tynan A. Bowel complications after radiotherapy for carcinoma of the prostate: the volume effect. Int J Radiat Oncol Biol Phys. 1990;18(2):315-320.

10. Hardcastle N, Davies A, Foo K, Miller A, Metcalfe PE. Rectal dose reduction with IMRT for prostate radiotherapy. J Med Imaging Radiat Oncol. 2010;54(3):235-248.

11. Ashman JB, Zelefsky MJ, Hunt MS, Leibel SA, Fuks Z. Whole pelvic radiotherapy for prostate cancer using 3D conformal and intensitymodulated radiotherapy. Int J Radiat Oncol Biol Phys. 2005;63(3): 765-771. 
12. Jani AB, Su A, Milano MT. Intensity-modulated versus conventional pelvic radiotherapy for prostate cancer: analysis of acute toxicity. Urology. 2006;67(1):147-151.

13. Luxton G, Hancock SL, Boyer AL. Dosimetry and radiobiologic model comparison of IMRT and 3D conformal radiotherapy in treatment of carcinoma of the prostate. Int J Radiat Oncol Biol Phys. 2004; 59(1):267-284.

14. Nutting CM, Convery DJ, Cosgrove VP, et al. Reduction of small and large bowel irradiation using an optimized intensity-modulated pelvic radiotherapy technique in patients with prostate cancer. Int $J$ Radiat Oncol Biol Phys. 2000;48(3):649-656.

15. Ray A, Sarkar B. Small bowel toxicity in pelvic radiotherapy for postoperative gynecological cancer: comparison between conformal radiotherapy and intensity modulated radiotherapy. Asia Pac J Clin Oncol. 2013;9(3):280-284.

16. Alongi F, Fiorino C, Cozzarini C, et al. IMRT significantly reduces acute toxicity of whole-pelvis irradiation in patients treated with postoperative adjuvant or salvage radiotherapy after radical prostatectomy. Radiother Oncol. 2009;93(2):207-212.

17. Fiorino C, Alongi F, Perna L, et al. Dose-volume relationships for acute bowel toxicity in patients treated with pelvic nodal irradiation for prostate cancer. Int J Radiat Oncol Biol Phys. 2009;75(1):29-35.

18. Perna L, Alongi F, Fiorino C, et al. Predictors of acute bowel toxicity in patients treated with IMRT whole pelvis irradiation after prostatectomy. Radiother Oncol. 2010;97(1):71-75.

19. Devisetty K, Mell LK, Salama JK, et al. A multi-institutional acute gastrointestinal toxicity analysis of anal cancer patients treated with concurrent intensity-modulated radiation therapy (IMRT) and chemotherapy. Radiother Oncol. 2009;93(2):298-301.

20. Crenn P, Messing B, Cynober L. Citrulline as a biomarker of intestinal failure due to enterocyte mass reduction. Clin Nutr. 2008;27(3): 328-339.

21. Crenn P, Vahedi K, Lavergne-Slove A, Cynober L, Matuchansky C, Messing B. Plasma citrulline: a marker of enterocyte mass in villous atrophy-associated small bowel disease. Gastroenterology. 2003;124(5): 1210-1219.

22. Lutgens LC, Deutz NE, Gueulette J, et al. Citrulline: a physiologic marker enabling quantitation and monitoring of epithelial radiationinduced small bowel damage. Int J Radiat Oncol Biol Phys. 2003;57(4): 1067-1074.

23. Onal C, Kotek A, Unal B, et al. Plasma citrulline levels predict intestinal toxicity in patients treated with pelvic radiotherapy. Acta Oncol. 2011;50(8):1167-1174.
24. Kestin L, Goldstein N, Vicini F, Yan D, Korman H, Martinez A. Treatment of prostate cancer with radiotherapy: should the entire seminal vesicles be included in the clinical target volume? Int J Radiat Oncol Biol Phys. 2002;54(3):686-697.

25. Sanguineti G, Little M, Endres EJ, Sormani MP, Parker BC. Comparison of three strategies to delineate the bowel for whole pelvis IMRT of prostate cancer. Radiother Oncol. 2008;88(1):95-101.

26. Roach M 3rd, Marquez C, You HS, et al. Predicting the risk of lymph node involvement using the pre-treatment prostate specific antigen and Gleason score in men with clinically localized prostate cancer. Int $J$ Radiat Oncol Biol Phys. 1994;28(1):33-37.

27. Deville C, Both S, Hwang WT, Tochner Z, Vapiwala N. Clinical toxicities and dosimetric parameters after whole-pelvis versus prostate-only intensity-modulated radiation therapy for prostate cancer. Int J Radiat Oncol Biol Phys. 2010;78(3):763-772.

28. Deville C, Vapiwala N, Hwang WT, et al. Comparative toxicity and dosimetric profile of whole-pelvis versus prostate bed-only intensitymodulated radiation therapy after prostatectomy. Int J Radiat Oncol Biol Phys. 2012;82(4):1389-1396.

29. Cheng JC, Schultheiss TE, Nguyen KH, Wong JY. Acute toxicity in definitive versus postprostatectomy image-guided radiotherapy for prostate cancer. Int J Radiat Oncol Biol Phys. 2008;71(2):351-357.

30. Brundage M, Sydes MR, Parulekar WR, et al. Impact of radiotherapy when added to androgen-deprivation therapy for locally advanced prostate cancer: long-term quality-of-life outcomes from the NCIC CTG PR3/MRC PR07 randomized trial. J Clin Oncol. 2015; 33(19):2151-2157.

31. Harris VA, Staffurth J, Naismith O, et al; PIVOTAL Trialists. Consensus guidelines and contouring atlas for pelvic node delineation in prostate and pelvic node intensity modulated radiation therapy. Int $J$ Radiat Oncol Biol Phys. 2015;92(4):874-883.

32. Crenn P, Coudray-Lucas C, Thuillier F, Cynober L, Messing B. Postabsorptive plasma citrulline concentration is a marker of absorptive enterocyte mass and intestinal failure in humans. Gastroenterology. 2000; 119(6):1496-1505.

33. Lutgens LC, Deutz N, Granzier-Peeters M, et al. Plasma citrulline concentration: a surrogate end point for radiation-induced mucosal atrophy of the small bowel. A feasibility study in 23 patients. Int $J$ Radiat Oncol Biol Phys. 2004;60(1):275-285.
OncoTargets and Therapy

\section{Publish your work in this journal}

OncoTargets and Therapy is an international, peer-reviewed, open access journal focusing on the pathological basis of all cancers, potential targets for therapy and treatment protocols employed to improve the management of cancer patients. The journal also focuses on the impact of management programs and new therapeutic agents and protocols on

\section{Dovepress}

patient perspectives such as quality of life, adherence and satisfaction. The manuscript management system is completely online and includes a very quick and fair peer-review system, which is all easy to use. Visit http://www.dovepress.com/testimonials.php to read real quotes from published authors. 